\title{
Serotonin 2A - I438 G/A and G-Protein Beta3 Subunit C825T Polymorphisms in Patients with Depression and SSRI-Associated Sexual Side-Effects
}

\author{
Jeffrey R Bishop', Jessica Moline ${ }^{2}$, Vicki L Ellingrod ${ }^{*, 2}$, Susan K Schultz ${ }^{3}$ and Anita H Clayton ${ }^{4}$ \\ 'University of Illinois at Chicago College of Pharmacy, Chicago, IL, USA; ${ }^{2}$ University of lowa College of Pharmacy, lowa City, IA, USA; ${ }^{3}$ Department \\ of Psychiatry, University of lowa Carver College of Medicine, lowa City, IA, USA; ${ }^{4}$ Department of Psychiatric Medicine, University of Virginia School \\ of Medicine, Charlottesville, VA, USA
}

\begin{abstract}
The occurrence of sexual side-effects from antidepressants is thought to be mediated through serotonin 2A (5HT2A) receptors. It is currently unknown if functional polymorphisms in the $5 \mathrm{HT} 2 \mathrm{~A}$ receptor or its $\mathrm{G}$-protein second messenger complex are related to sexual dysfunction in patients taking an selective serotonin reuptake inhibitor (SSRI) for depression. The purpose of this study was to determine the relationship of the 5HT2A - I438 G/A and GNB3 C825T single nucleotide polymorphisms with overall sexual well-being and individual components of sexual health as measured by the Changes in Sexual Functioning Questionnaire (CSFQ). We evaluated 89 outpatients ( $18-40$ years of age) at low risk for other causes of sexual dysfunction who were being treated for depression with an SSRI and did not have sexual difficulties before taking the antidepressant. Outcome measures were stratified by 5HT2A and GNB3 genotypes. After controlling for age, gender, anxiety scale scores, and depression scale scores, persons with a GG genotype of the 5HT2A - I438 single nucleotide polymorphisms (SNP) were significantly more likely to be categorized as having sexual dysfunction than persons with a GA or AA genotype $(\mathrm{OR}=3.6 ; 95 \% \mathrm{Cl}$ I.03, I2.6; $p=0.046)$. Furthermore, the 5HT2A - $1438 \mathrm{GG}$ genotype was a significant predictor of lower arousal scores $(p=0.022)$ after accounting for other measures. There was no significant relationship between any outcome measure and GNB3 genotype.

Neuropsychopharmacology (2006) 31, 228 I-2288. doi:I0.1038/sj.npp. I 30 I090; published online 10 May 2006
\end{abstract}

Keywords: sexual side-effects; SSRI; depression; serotonin 2A receptor; G-protein beta subunit; pharmacogenetics

\section{INTRODUCTION}

Sexual dysfunction is a common and disconcerting sideeffect of SSRIs that influences a patient's desire to continue long-term antidepressant treatment (Bull et al, 2002; Clayton et al, 2002; Montejo et al, 2001). Despite clinical study reports summarized in prescriber information sheets indicating the emergence of sexual difficulties in $<15 \%$ of subjects, most investigations of this topic show that the real incidence of sexual side-effects subsequent to SSRI initiation is between 20 and $70 \%$ (Clayton et al, 2002; Montejo-Gonzalez et al, 1997; Montejo et al, 1996, 2001). Sexual side-effects from these medications are of significant clinical importance, particularly in young, sexually active

\footnotetext{
*Correspondence: Dr VL Ellingrod, University of lowa College of Pharmacy, Clinical and Administrative Division, S4 13 Pharmacy Building, lowa City, IA 52242, USA, Tel: + I 319335 8837, Fax: + I 319353 5646, E-mail: vicki-ellingrod@uiowa.edu

Received 20 December 2005; revised I March 2006; accepted 27 March 2006

Online publication: 28 March 2006 at http://www.acnp.org/citations/ Npp032806050742/default.pdf
}

patients. Previous research has shown that approximately $40 \%$ of persons starting an SSRI will either discontinue or switch medications owing to an adverse event (Bull et al, 2002). Of persons discontinuing or switching SSRIs due to adverse events, over one-third listed sexual side-effects as either primary or secondary reasons for treatment cessation (Bull et al, 2002). In another study of over 1000 outpatients followed prospectively after antidepressant initiation, nearly $40 \%$ of persons experiencing changes in their sexual wellbeing indicated that it was sufficient enough to warrant medication discontinuation (Montejo et al, 2001).

There are a number of variables associated with the development of sexual dysfunction. Substance use/abuse, smoking, marital status, poor health, medication use, and other disease states have all been shown to have a relationship with sexual well-being in either depressed or euthymic individuals (Clayton et al, 2002; Laumann et al, 1999). The serotonergic actions of SSRIs have been implicated in the development of sexual side-effects (Murray, 1998). Pharmacologically, these medications inhibit the serotonin transporter (5HTT) (Stahl, 1998) and increase the synaptic latency period of serotonin $(5 \mathrm{HT})$ molecules and their 
abilities to agonize post-synaptic serotonin receptors. There are many types of post-synaptic serotonin receptors, some of which are reported to be responsible for increases in mood and decreases in anxiety (eg 5HT1A), whereas others have been shown to be related to side-effects from SSRIs (eg 5HT2A receptors). Animal research has shown that action through the 5HT2A subclass of serotonin receptors decreases the sexual arousal and ejaculation components of the sexual process (Bitran and Hull, 1987). Furthermore, antidepressants such as nefazodone that block 5HT2A receptors are not associated with the significant development of sexual side-effects (Clayton et al, 2002), and 5HT2A antagonists have been shown to reverse SSRI-associated sexual side-effects in some studies (Aizenberg et al, 1995, 1997).

Genetic variability in the $5 H T 2 A$ promoter $(-1438 \mathrm{G} / \mathrm{A})$, as well as the beta subunit of its G-protein second messenger (GNB3 C825T) may have functional consequences that may affect serotonergic transmission through this receptor (Levine et al, 1990; Parsons et al, 2004). Recent analysis of the differential expression of the -1438 SNP has suggested that the AA genotype is associated with higher 5HT2A gene expression in cell lines also endogenously expressing 5HT2A (Parsons et al, 2004). Another investigation published by Murphy and colleagues (Murphy et al, 2003) showed that the silent 5HT2A (T102C) SNP in linkage disequilibrium with the -1438 polymorphism was significantly related to antidepressant intolerance in elderly subjects treated with paroxetine. The GNB3T allele is associated with increased G-protein second messenger activity, as well as hypertension (Pietruck et al, 1996; Siffert et al, 1995, 1999).

To our knowledge, the relationship between sequence variability in these pharmacogenetic candidate genes and sexual side-effects has not been studied. Therefore, the purpose of this pilot investigation was to examine the 5HT2A - $1438 \mathrm{G} / \mathrm{A}$ and GNB3 C825T SNPs and their relationship to overall sexual well-being, or individual components of sexual health (eg arousal, interest, orgasm, interest, desire/frequency, or general interest) as measured by the CSFQ in a population of 18 to 40 -year-old persons at low risk for other causes of sexual dysfunction.

\section{MATERIALS AND METHODS}

\section{Subjects}

Eighty-nine outpatients with depression being treated with an SSRI were recruited for this point prevalence study. Subjects were recruited through local advertisements for a study of 'health and well-being' while being treated with citalopram, escitalopram, fluoxetine, paroxetine, or sertraline for depression. Potential research subjects underwent telephone screenings where they were assessed for eligibility and informed that the study would consist of an interview session to determine the efficacy of current antidepressant therapy, cheek swab for DNA sample collection, and questionnaire to assess changes in sexual well-being while being treated with an SSRI.

Subjects were included if they had been treated with medication for at least 6 weeks, were between the ages of 18 and 40, and did not have problems with sexual desire or functioning before beginning medication. Potential subjects were excluded if they were taking another medication for depression or any other medication known to affect sexual functioning (either positively or negatively), if they had any other documented primary Axis I diagnosis, cardiovascular disease, neurological disorder, diabetes mellitus (Type I or II), genitourinary disease, or reported frequent urinary tract infections. Persons who scored $>10$ on the HAM-D assessment were excluded from our data analysis to minimize the confounding effect of depression on sexual well-being scores. This resulted in 81 subjects. Chart reviews were done to confirm that subjects met the above entry/inclusion criteria and had a diagnosis of depression.

\section{Assessments}

Subjects were assessed in person at the University of Iowa General Clinical Research Center where they were consented for the study, using an informed consent document approved by the University of Iowa Institutional Review Board. General assessments included vital signs, height, weight, and sociodemographic variables previously associated with sexual well-being (ie age, race/ethnicity, marital status, number of children, years of education, alcohol consumption, smoking status, or illicit drug use). Assessments of depression status included the Hamilton Rating Scale for Depression 21 item version (HAM-D), Hamilton Rating Scale for Anxiety (HAM-A), specific SSRI used, dose, and duration of use. Dose categories were designed to evaluate the effect of medication dose on sexual dysfunction. For the purposes of this investigation, 'higher' doses of SSRI medication were defined as $\geqslant 40 \mathrm{mg} /$ day of citalopram, fluoxetine, or paroxetine; $\geqslant 100 \mathrm{mg} /$ day of sertraline, and $\geqslant 20 \mathrm{mg} /$ day escitalopram. Dosing characteristics for the study population are included in Table 1 . Two of the authors (Jeffrey R Bishop and Jessica Moline) conducted all of the assessments and interviews. At the beginning of the study, these two trained raters simultaneously assessed subjects with the HAM-D and HAM-A and formed consensus scores until 10 assessments in a row were done with scores within one point of each other to assure inter-rater reliability.

Sexual dysfunction was assessed with the self-administered CSFQ (Clayton et al, 1997a). The CSFQ consists of separate assessments for males and females, and has been validated in healthy and depressed clinic populations, and used in both longitudinal and cross sectional studies of sexual well-being (Clayton et al, 1995, 1997b, 2002; Keller et al, 2006). Questions on the CSFQ assess a variety of

Table I Antidepressant Dosing

\begin{tabular}{lcc}
\hline Medication & Mean dose (SD) & Dose range (mg/day) \\
\hline Citalopram & $33.3(9.85)$ & $20-40$ \\
Escitalopram & $13.04(4.46)$ & $10-20$ \\
Fluoxetine & $28.13(15.15)$ & $10-60$ \\
Paroxetine & $25(11.18)$ & $20-45$ \\
Sertraline & $74(35.7 \mid)$ & $50-200$ \\
\hline
\end{tabular}

Antidepressant dose characteristics (reported in $\mathrm{mg} /$ day) for the study population as a whole. 
different causes of change in sexual functioning or desire (eg relationship changes, stress level, illness, medications, etc). Sexual dysfunction is determined by falling below thresholds (47 for males and 41 females on a scale of 14-70), where lower scores are indicative of decreased sexual desire or functioning. The CSFQ also consists of subscales to assess specific aspects of sexual well-being. Pleasure (scored 1 to 5), desire/interest (scored 3 to 15), desire/frequency (scored 2 to 10), arousal (scored 3 to 15), and orgasm (scored 3 to 15 ) subscales are all assessed by questions on the CSFQ.

\section{Genotyping}

Genomic DNA was isolated from buccal cells collected with cheek brushes (Cyto-pak, Medical Packaging Corp, Camarillo, CA) with a previously described protocol (Richards et al, 1993). PCR primers used for the 5HT2A -1438G/A polymorphism (GENBANK AL160397) have been utilized in previous investigations of this pharmacogenetic candidate gene (Arranz et al, 1998). Forty-five PCR cycles were done for reactions in a $30 \mu \mathrm{l}$ volume with $1.5 \mathrm{mM} \mathrm{Mg}^{2+}$ and $10 \mathrm{pmol}$ of each primer according to the following specifications: initial denaturation for $5 \mathrm{~min}$ at $94^{\circ} \mathrm{C}, 94^{\circ} \mathrm{C}$ for $30 \mathrm{~s}, 56^{\circ} \mathrm{C}$ for $30 \mathrm{~s}, 72^{\circ} \mathrm{C}$ for $30 \mathrm{~s}$, and a final extension at $72^{\circ} \mathrm{C}$ for $10 \mathrm{~min}$. The 468 -bp product was digested with $\mathrm{MspI}$ and visualized on a $10 \%$ polyacrylamide gel stained with ethidium bromide. Sequences with a G allele were digested into fragments of 244 and $224 \mathrm{bp}$ whereas those carrying an A allele remained uncut.

PCR and sequencing primers for the C825T polymorphism in exon 10 of the GNB3 gene (GENBANK U47924) were designed using Pyrosequencing SNP Primer Design Version 1.01 software (http://www.pyrosequencing.com). PCR reactions were done in a $30 \mu \mathrm{l}$ volume with $1.5 \mathrm{mM} \mathrm{Mg}^{2+}$, 10 pmol of forward primer (5'-CTCCCACGAGAGCATCA TC), and $12 \mathrm{pmol}$ of reverse primer ( $5^{\prime}$-TGCAGTTGAAGTC GTCGTAGC) with the same cycle specifications as those outlined above for the 5HT2A - 1438 polymorphism. Genotyping for the C825T SNP was done with Pyrosequencing $^{\mathrm{TM}}$ Technology (Ronaghi, 2003). PCR products were visualized by electrophoresis on $1.8 \%$ agarose gels stained with ethidium bromide before Pyrosequencing. The sequencing primer used was $5^{\prime}$-CTGCGGCATCACGTC.

\section{Data Analysis}

Differences in sociodemographic variables between medication categories and genotype groups were determined with the $\chi^{2}$ or student's $t$-test/one-way analysis of variance for dichotomous and normally distributed continuous variables, respectively. It was determined a priori that only persons without clinically significant depressive symptoms (eg HAM-D scores $\leqslant 10$ ) would be included in this analysis and that the primary genetic analyses would consist of conducting a basic allelic association study of $5 H T 2 A-1438$ G/A and GNB3 $825 \mathrm{C} / \mathrm{T}$ SNPs with sexual dysfunction as determined by the CSFQ total score, followed by genotypespecific analyses of the -1438 GG and 825 TT SNPs for their relationship to CSFQ outcomes. Overall sexual dysfunction associated with SSRI treatment was examined as a categorical variable as previously described (Clayton et al, 1997a), whereas sub-scale measures (eg pleasure, frequency, interest, arousal, and orgasm) were all assessed as continuous variables. Subscale measures were stratified by gender.

Spearman's Rho was used to look for potential confounders between variables. Univariate logistic regression analyses, Wald $\chi^{2}$ test statistics, and 95\% confidence intervals were done to determine the associations of clinical and genetic variables to sexual dysfunction. Subsequent separate multiple logistic regression analyses were done for 5HT2A and GNB3 SNPs to control for other relevant variables. For the 5HT2A - 1438 GG and GNB3 TT genotypes, additional variables in the regression analyses included age, gender, HAM-D scores, and HAM-A scores.

$T$-tests were used to assess differences in the normally distributed CSFQ subscale measures by genotype group. A subsequent linear regression analysis was done to assess the relationship between the arousal sub-scale measure and the 5HT2A GG genotype after controlling for age, gender, HAM-D scores, and HAM-A scores.

A two-tailed alpha value of $\leqslant 0.05$ was used as the threshold for statistical significance. SAS version 9.0 and SAS JMP version 5.01a (SAS Institute Inc., Cary, NC, 2002) were used for all statistical analyses.

\section{RESULTS}

Eighty-nine subjects were enroled in this study. Follow up chart reviews revealed four persons with medical conditions or medications that excluded them from the study. To minimize the confounding effects of depressive symptoms, only persons not exhibiting clinically significant depressive symptoms (eg HAM-D $\leqslant 10$ ) were included in the data analysis. The resulting study population included in this analysis consisted of 81 participants, $75 \%$ of whom were Caucasian females (see Table 1). Thirty-five of the 81 self-administered questionnaires analyzed were not completely filled out by participants. The results of the self administered CSFQ allowed us to categorize whether a subject had sexual dysfunction associated with SSRI treatment in $68 / 81(84 \%)$ of respondents. Forty-six out of 81 volunteers (57\%) completed all data for the Pleasure and Interest CSFQ subscale measures, 81/81 (100\%) completed all data for the Frequency and Arousal CSFQ subscales, and $79 / 81(98 \%)$ completed all questions pertaining to the Orgasm subscale measure.

Overall, the prevalence of SSRI-associated sexual dysfunction in this population was $31 \%$, with a rate of $35 \%$ in females and $18 \%$ in males. There were no differences in sexual dysfunction (as measured by failing to exceed the gender-specific threshold on the Total score), or any CSFQ subscale measure between SSRI medication groups in either the whole population or in gender subgroups (see Table 2). Fifty-one $(63 \%)$ of the volunteers in this study were receiving a 'lower' dose of SSRI. There were no differences in CSFQ outcomes (total score or any subscale measure), anxiety, or depression scale scores between persons receiving a 'higher' or 'lower' dose of SSRI.

Genotype and allele distributions for the 5HT2A-1438 G/A and GNB3 $825 \mathrm{C} / \mathrm{T}$ SNPs are presented in Table 1. The allele frequencies for both candidate gene loci were in Hardy-Weinberg equilibrium $\left(\chi^{2}=0.02\right.$, 1df, $p=0.90$ for 
Table 2 Subject Demographics and Characteristics Stratified by Genotype

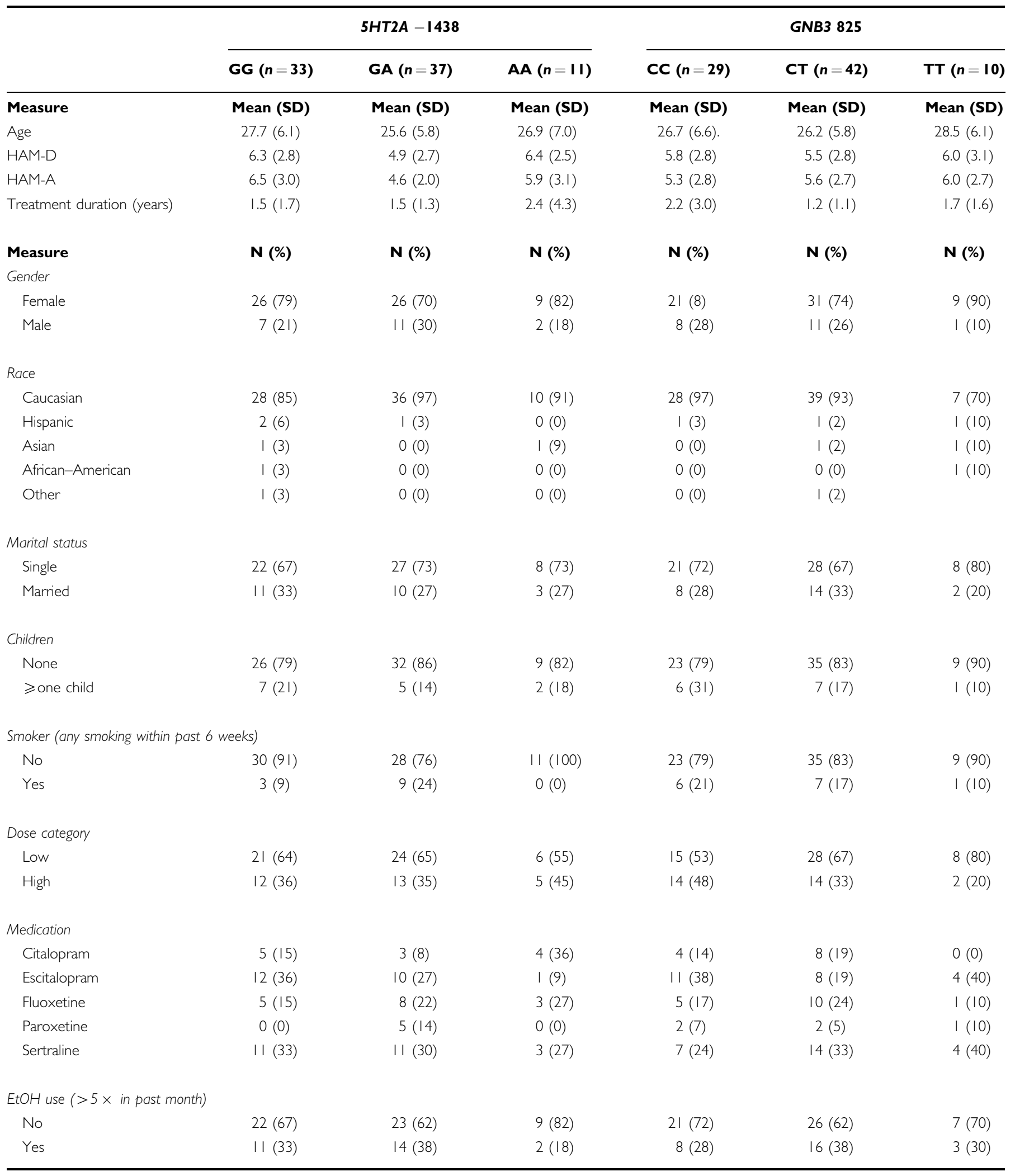

HAM-D, Hamilton Rating Scale for Depression 21 item version; HAM-A, Hamilton Rating Scale for Anxiety.

5HT2A and $\chi^{2}=0.77,1 \mathrm{df}, p=0.38$ for GNB3), with variant alleles $(-1438 \mathrm{~A}$ and $825 \mathrm{~T})$ being common in this population (frequencies of 0.36 and 0.38 , respectively). No statistically significant differences between genotype groups were observed for gender, age, race, marital status, smoking status, dose category, or ethanol consumption variables. 
Table 3 Analysis of CSFQ Outcomes by Gender and 5HT2A - I438 Genotype

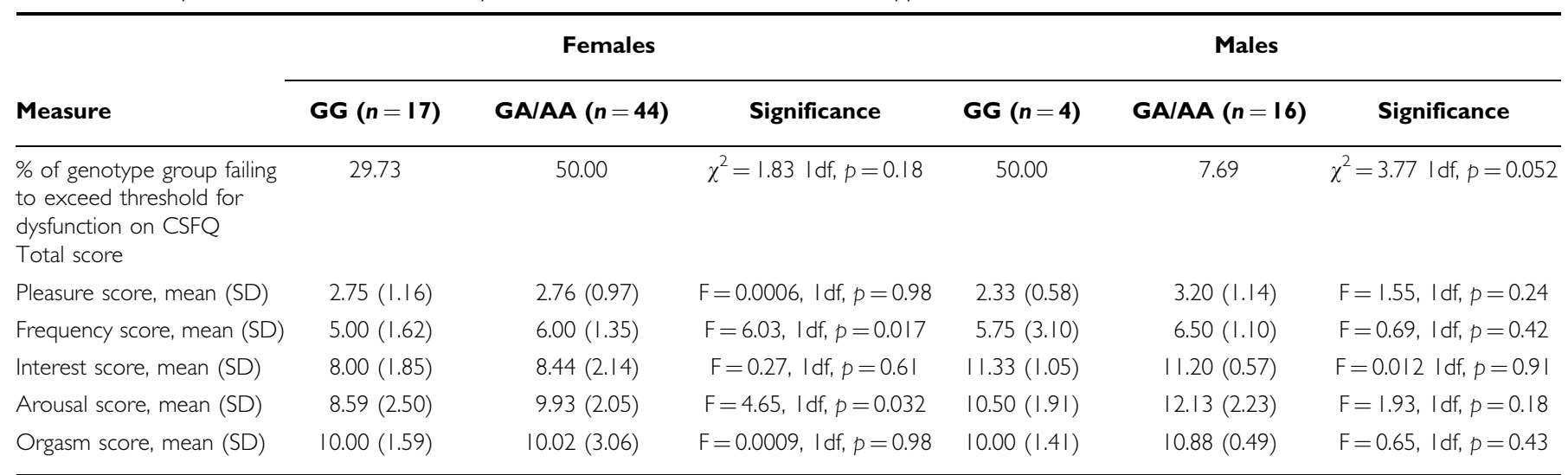

CSFQ, changes in sexual functioning questionnaire; SD, standard deviation.

Table 3 includes CSFQ outcome measures stratified by genotype and gender for individuals completing the respective measures on the self-administered questionnaire.

The presence of a G allele of the $5 H T 2 A-1438$ SNP was not significantly associated with sexual dysfunction in this sample $\left(\chi^{2}=0.002\right.$, $\left.1 \mathrm{df}, p=0.97\right)$. However, the 5HT2A GG genotype was significantly associated with persons being categorized as having sexual dysfunction, as measured by overall CSFQ score $\left(\chi^{2}=4.19,1 \mathrm{df}, p=0.041\right)$. No statistically significant differences in gender, age, BMI, treatment duration, smoking rate, or alcohol/substance use $(>5 \times$ in the past month) were seen between 5HT2A - 1438 genotype groups. Anxiety rating scale scores were significantly higher in the GG group compared to GA/AA subjects (6.8 vs 5.1, respectively; $p=0.014)$. After controlling for age, gender, and anxiety and depression scale scores, persons with a GG genotype of the $5 H T 2 A-1438$ SNP were significantly more likely to be categorized as having sexual dysfunction than persons with a $\mathrm{GA}$ or AA genotype (OR $=3.6 ; 95 \%$ CI $1.03,12.6 ; p=0.046)$.

The presence of any T allele of the GNB3 825 SNP trended toward a significant association with sexual dysfunction $\left(\chi^{2}=3.60\right.$, 1df, $\left.p=0.058\right)$. Further analysis showed that the GNB3 TT genotype was not associated with persons falling into the sexual dysfunction category of the overall CSFQ scale score $\left(\chi^{2}=2.0,1 \mathrm{df}, p=0.16\right)$. No statistically significant differences in gender, age, treatment duration, smoking rate, alcohol/substance use, anxiety scale scores, or depression scale scores were observed between persons with a TT or TC genotype and CC individuals. After controlling for age, gender, anxiety, and depression scale scores, the GNB3 TT genotype was still not significantly associated with an increased odds of having sexual dysfunction in this population ( $\mathrm{OR}=1.34 ; 95 \% \mathrm{CI} 0.55,10.4 ; p=0.25)$.

The analysis of CSFQ outcomes stratified by gender and genotype are summarized in Table 3. Arousal subscale scores were significantly lower in females with a 5HT2A -1438 GG genotype $($ mean $=8.6 \pm 2.5)$ compared to GA/AA $(9.9 \pm 2.05)$ individuals $(\mathrm{F}=4.6,1 \mathrm{df}, p=0.032)$ on a scale of three to 15 . These values were not statistically different in males (mean $=10.5 \pm 1.9$ for the GG group $v s 12.1 \pm 2.1$ for the GA/AA subjects; $F=5.0,1 \mathrm{df}, p=0.18)$. Desire/frequency subscale scores followed a similar trend as the arousal measure and were significantly lower in females with a $5 H T 2 A$ -1438 GG genotype $($ mean $=5.0 \pm 1.62)$ compared to GA/AA $(6 \pm 1.35)$ individuals $(\mathrm{F}=4.6,1 \mathrm{df}, p=0.04)$ on a scale of 2 to 10 . These values were not statistically different in males, but followed a similar numerical trend (mean $=$ $5.8 \pm 3.1$ for the GG group $v s 6.5 \pm 1.1$ for the GA/AA subjects; $\mathrm{F}=0.69,1 \mathrm{df}, p=0.42$ ). No differences in pleasure, interest, desire, or orgasm subscales existed between 5 HT2A genotype groups for either gender group. No significant differences in CSFQ subscale measures were seen between GNB3 genotype groups.

A linear regression analysis was done to assess the relationship between 5 HT2A genotypes and the arousal CSFQ subscale measure after accounting for other clinically relevant variables. The GG genotype alone was significantly predictive of lower arousal scores $(\mathrm{F}=7.03,1 \mathrm{df}, p=0.0097)$. After controlling for age, gender, anxiety scale scores, and depression scale scores, the 5HT2A - 1438 GG genotype was still a significant predictor of lower arousal scores $(p=0.022)$. This model collectively predicted $25 \%$ of the variability in arousal subscale measures $(\mathrm{F}=5.10,5 \mathrm{df}, p=0.0004)$.

\section{DISCUSSION}

The primary result of this study is that the 5 HT2A -1438 GG genotype was significantly associated with sexual dysfunction seen in the context of SSRI therapy. This finding was observed in subjects who were no longer clinically depressed, and who had not experienced sexual difficulties before initiation of antidepressant therapy. Further analysis of the different aspects of sexual well-being assessed by the validated CSFQ utilized in our study suggests that this may primarily be a function of decreased levels of sexual arousal in persons with this genotype. Although statistical significance between genotype groups for the arousal sub-scale measure was only seen in females, the arousal score differential between genotype groups in males was very similar. It is possible that the relatively small number of males that participated in this study could have resulted in a Type II error, preventing us from finding statistical significance in this gender group. The numerical differences between genotype groups in females translate to approximate arousal levels (frequency of arousal, ease of arousal, and achieving adequate lubrication) as occurring 'more than $\frac{1}{2}$ of 
the time' for the AA/AG group vs between 'less than $\frac{1}{2}$ the time' and 'much less than $\frac{1}{2}$ of the time' for the GG group. Differences in values for males translate to approximate arousal levels (frequency of arousal, ease of arousal, and ease of ability to achieve an erection) as occurring 'much more than $\frac{1}{2}$ of the time' for the AA/AG group $v s$ between 'much less than $\frac{1}{2}$ of the time' and 'about $\frac{1}{2}$ of the time' for the GG group. Therefore, these differences appear to be clinically significant. Furthermore, after controlling for other clinically relevant variables found to affect sexual well-being in previous studies (eg age, gender, and anxiety/ depression scale scores), the GG genotype was significantly predictive of lower arousal scores.

The results reported here correlate with those previously presented by Murphy et al (Murphy et al, 2003) who showed that the 102CC genotype (in complete linkage disequilibrium (LD) with the -1438 GG SNP) was significantly related to treatment discontinuation in an elderly patient population treated with paroxetine. This effect was not seen in those patients treated with mirtazapine, an antidepressant agent with 5HT2A antagonistic activities. The primary outcome for that analysis was treatment discontinuation, which collectively resulted from a variety of reasons, including gastrointestinal upset, difficulty concentrating, agitation, sleep disturbance, dizziness, sweating, headache, and sexual dysfunction. Medication discontinuation in the 102 CC group was over two times that in the TC/TT group. Our results confirm the heightened sensitivity to adverse effects seen in persons with this genotype.

A number of previous investigations have discussed the relative effects of serotonergic agents, such as SSRIs, on the different aspects of sexual well-being (Kennedy et al, 2000; Montejo et al, 2001; Rosen et al, 1999). Notably, the most clinically significant effects seem to be on arousal in women and orgasm in men (Clayton et al, May 2003). We did not observe a genetic relationship with the orgasm subscale measure in this population. However, we found a significant genotype relationship with the desire/frequency subscale measure of the CSFQ. After further investigation, it was determined that the arousal and desire/frequency subscales were highly correlated, and that arousal scores significantly predicted desire/frequency scores $\left(R^{2}=0.35, \mathrm{~F}=43,1 \mathrm{df}\right.$, $p<0.0001)$. It makes sense that a person who is not sexually aroused would participate in sexual activities less, while the opposite relationship (ie low frequency predicting decreased arousal) does not seem quite as plausible.

There are other pharmacogenetic candidate genes that may be relevant to investigate in the context of SSRIassociated side-effects, two of which have been implicated in SSRI response. Functional variability within the serotonin transporter promoter region has been shown to affect transcription of this gene (Lesch et al, 1996). The resulting changes in serotonin re-uptake from the synaptic cleft could influence activity at serotonin receptors and therefore SSRI tolerability. It is also possible that Tryptophan hydroxylase (TPH) variants may differentially affect an individual's tolerability to an SSRI. Tryptophan hydroxylase is the enzyme responsible for the conversion of TPH to 5-hydroxy tryptophan $(5 \mathrm{OH}-\mathrm{TPH}$, which is then decarboxylated to form serotonin. The TPH to $5 \mathrm{OH}-\mathrm{TPH}$ conversion the ratelimiting step in the conversion of serotonin, and polymorphisms have been found in both the TPH1 and TPH2 genes (Breidenthal et al, 2004; Nielsen et al, 1997). Evidence is inconsistent for the involvement of a common TPH1 SNP (A218C) in SSRI treatment response (Serretti et al, 2001; Yoshida et al, 2002) with no studies to date done to investigate treatment response and TPH2, which has been argued to be responsible for 5HT formation in the brain (Shaltiel et al, 2005). Certainly, other genes with noted SNPs may play a role in SSRI tolerability in the context of sexual dysfunction (eg monoamine oxidase A or nitric oxide synthase). These and possibly other candidates were not examined in our study and should be included in future analyses of SSRI-associated side-effects.

There are a number of limitations that are relevant to mention in the context of this study. This was a point prevalence analysis that utilized a questionnaire to determine changes in sexual functioning associated with drug utilization. Arguably, a prospective analysis would be better suited to study sexual dysfunction as a treatment-emergent effect. This being said, sexual dysfunction is known to be one of the few persistent side-effects associated with SSRI treatment, with over $85-90 \%$ of persons who experience this side effect not having resolution after 6 months of treatment (Ashton and Rosen, 1998; Montejo-Gonzalez et al, 1997). In an attempt to minimize the confounding effects of residual depressive symptoms on sexual functioning, we enrolled subjects who had been on SSRIs for at least 6 weeks, in order for them to have had an adequate chance to respond to medication. While this was obviously achieved in our study (mean HAM-D scores = 5.7 in the subjects analyzed), we may have arguably missed those subjects who discontinued an SSRI before 6 weeks because of sexual side-effects. It is our opinion that missing these subjects is more likely to have diluted the effect that was seen in this sample, rather than resulting in a spuriously positive outcome. It should also be noted that while we required a minimum of 6 weeks SSRI treatment for study entry, the treatment duration was quite variable in our population $(1.65 \pm 2.06$ years $)$. It is possible that some of the subjects may have had a gradual improvement in their sexual functioning over time, which could have also lowered the point prevalence sexual dysfunction values observed and possibly minimized the pharmacogenetic relationship seen. Finally, our sample was heavily weighted in favor of females over males, which affects our ability to make firm conclusions about this genetic relationship among male patients. Future investigations involving more participants (particularly males) will be needed to confirm these results.

Despite these limitations, the present study is a significant contribution to the psychiatric pharmacogenetics literature. Sexual dysfunction is arguably the most clinically significant SSRI-related adverse event in the age group studied here (18-40 years). Furthermore, we utilized a previously validated assessment to determine not only the overall measure of sexual health in these subjects, but also the different aspects of sexual well-being. This resulted in being able to determine an enhanced relationship between sexual arousal and the -1438 GG genotype. Our subject population was almost completely devoid of confounding medical and pharmacological confounders, which is extremely rare for this type of investigation. Many studies analyzing sexual dysfunction related to antidepressant therapy do not exclude persons based on concurrent medications or other 
diagnoses. Although that strategy increases the generalizability of those types of studies, employing that methodology in the current study would have made it extremely difficult to determine the relative effects of genetics and medical variables that play a role in a person's overall state of sexual well-being.

In conclusion, we report that in persons taking an SSRI for depression, but who were not clinically depressed or anxious, individuals with the 5HT2A - 1438 GG genotype were over 3.5 times more likely to have sexual dysfunction associated with antidepressant use than those without this genotype. Further analysis suggests that this may be a function of persons with a GG genotype having lower sexual arousal scores, particularly in females taking an SSRI. Future prospective analyses of this phenomenon in reproductive-aged patients being treated with these agents for depression will allow us to determine the utility of using this genetic marker as one mechanism for screening patients prior to antidepressant use to individualize antidepressant medication regimens.

\section{ACKNOWLEDGEMENTS}

This work was supported by grant RR00059 from the General Clinical Research Centers Program, National Center for Research Resources, grant K08MH64158-02 from the National Institute of Mental Health, and was completed while Dr Bishop was an Iowa Scholars in Clinical Investigation Program K30 trainee (K30HL04117-01A1).

\section{REFERENCES}

Aizenberg D, Gur S, Zemishlany Z, Granek M, Jeczmien P, Weizman A (1997). Mianserin, a 5-HT2a/2c and alpha 2 antagonist, in the treatment of sexual dysfunction induced by serotonin reuptake inhibitors. Clin Neuropharmacol 20: 210-214.

Aizenberg D, Zemishlany Z, Weizman A (1995). Cyproheptadine treatment of sexual dysfunction induced by serotonin reuptake inhibitors. Clin Neuropharmacol 18: 320-324.

Arranz MJ, Munro J, Owen MJ, Spurlock G, Sham PC, Zhao J et al (1998). Evidence for association between polymorphisms in the promoter and coding regions of the 5-HT2A receptor gene and response to clozapine. Mol Psychiatry 3: 61-66.

Ashton AK, Rosen RC (1998). Accommodation to serotonin reuptake inhibitor-induced sexual dysfunction. J Sex Marital Ther 24: 191-192.

Bitran D, Hull EM (1987). Pharmacological analysis of male rat sexual behavior. Neurosci Biobehav Rev 11: 365-389.

Breidenthal SE, White DJ, Glatt CE (2004). Identification of genetic variants in the neuronal form of tryptophan hydroxylase (TPH2). Psychiatr Genet 14: 69-72.

Bull SA, Hunkeler EM, Lee JY, Rowland CR, Williamson TE, Schwab JR et al (2002). Discontinuing or switching selective serotonin-reuptake inhibitors. Ann Pharmacother 36: 578-584.

Clayton AH, McGarvey EL, Clavet GJ (1997a). The Changes in Sexual Functioning Questionnaire (CSFQ): development, reliability, and validity. Psychopharmacol Bull 33: 731-745.

Clayton AH, McGarvey EL, Clavet GJ, Piazza L (1997b). Comparison of sexual functioning in clinical and nonclinical populations using the Changes in Sexual Functioning Questionnaire (CSFQ). Psychopharmacol Bull 33: 747-753.

Clayton AH, McGarvey E, Keller A, Pinkerton R (May 2003). Burden of sexual dysfunction with antidepressant treatments. Abstracts of the 156th Annual Psychiatric Association Meeting.
Clayton AH, Owens JE, McGarvey EL (1995). Assessment of paroxetine-induced sexual dysfunction using the Changes in Sexual Functioning Questionnaire. Psychopharmacol Bull 31: 397-413.

Clayton AH, Pradko JF, Croft HA, Montano CB, Leadbetter RA, Bolden-Watson C et al (2002). Prevalence of sexual dysfunction among newer antidepressants. J Clin Psychiatry 63: 357-366.

Keller A, McGarvey EL, Clayton AH (2006). Reliability and construct validity of the Changes in Sexual Functioning Questionnaire short form (CSFQ-14). J Sex Marital Ther 32: 43-52.

Kennedy SH, Eisfeld BS, Dickens SE, Bacchiochi JR, Bagby RM (2000). Antidepressant-induced sexual dysfunction during treatment with moclobemide, paroxetine, sertraline, and venlafaxine. J Clin Psychiatry 61: 276-281.

Laumann EO, Paik A, Rosen RC (1999). Sexual dysfunction in the United States: prevalence and predictors. JAMA 281: 537-544.

Lesch KP, Bengel D, Heils A, Sabol SZ, Greenberg BD, Petri S et al (1996). Association of anxiety-related traits with a polymorphism in the serotonin transporter gene regulatory region. Science 274: 1527-1531.

Levine MA, Smallwood PM, Moen Jr PT, Helman LJ, Ahn TG (1990). Molecular cloning of beta 3 subunit, a third form of the G protein beta-subunit polypeptide. Proc Natl Acad Sci USA 87: 2329-2333.

Montejo AI, Llorca G, Izquierdo JA, Ledesma A, Bousono M, Calcedo A et al (1996). Sexual dysfunction secondary to SSRIs. A comparative analysis in 308 patients. Actas Luso Esp Neurol Psiquiatr Cienc Afines 24: 311-321.

Montejo AL, Llorca G, Izquierdo JA, Rico-Villademoros F (2001). Incidence of sexual dysfunction associated with antidepressant agents: a prospective multicenter study of 1022 outpatients. Spanish Working Group for the Study of Psychotropic-Related Sexual Dysfunction [see comment]. J Clin Psychiatry 62(Suppl 3): 10-21.

Montejo-Gonzalez AL, Llorca G, Izquierdo JA, Ledesma A, Bousono M, Calcedo A et al (1997). SSRI-induced sexual dysfunction: fluoxetine, paroxetine, sertraline, and fluvoxamine in a prospective, multicenter, and descriptive clinical study of 344 patients. J Sex Marital Ther 23: 176-194.

Murphy Jr GM, Kremer C, Rodrigues HE, Schatzberg AF (2003). Pharmacogenetics of antidepressant medication intolerance. Am J Psychiatry 160: 1830-1835.

Murray JB (1998). Physiological mechanisms of sexual dysfunction side effects associated with antidepressant medication. J Psychol 132: 407-416.

Nielsen DA, Jenkins GL, Stefanisko KM, Jefferson KK, Goldman D (1997). Sequence, splice site and population frequency distribution analyses of the polymorphic human tryptophan hydroxylase intron 7. Brain Res Mol Brain Res 45: 145-148.

Parsons MJ, D'Souza UM, Arranz MJ, Kerwin RW, Makoff AJ (2004). The $-1438 \mathrm{~A} / \mathrm{G}$ polymorphism in the 5-hydroxytryptamine type $2 \mathrm{~A}$ receptor gene affects promoter activity. Biol Psychiatry 56: 406-410.

Pietruck F, Moritz A, Montemurro M, Sell A, Busch S, Rosskopf D et al (1996). Selectively enhanced cellular signaling by $\mathrm{Gi}$ proteins in essential hypertension. G alpha i2, G alpha i3, G beta 1, and G beta 2 are not mutated. Circ Res 79: 974-983.

Richards B, Skoletsky J, Shuber AP, Balfour R, Stern RC, Dorkin HL et al (1993). Multiplex PCR amplification from the CFTR gene using DNA prepared from buccal brushes/swabs. Hum Mol Genet 2: 159-163.

Ronaghi M (2003). Pyrosequencing for SNP genotyping. Methods Mol Biol 212: 189-195.

Rosen RC, Lane RM, Menza M (1999). Effects of SSRIs on sexual function: a critical review [see comment]. J Clin Psychopharmacol 19: 67-85.

Serretti A, Zanardi R, Cusin C, Rossini D, Lorenzi C, Smeraldi E (2001). Tryptophan hydroxylase gene associated with paroxetine antidepressant activity. Eur Neuropsychopharmacol 11: 375-380. 
Shaltiel G, Shamir A, Agam G, Belmaker RH (2005). Only tryptophan hydroxylase (TPH)-2 is relevant to the CNS. Am J Med Genet B Neuropsychiatr Genet 136: 106.

Siffert W, Rosskopf D, Erbel R, Ritz E (1999). G protein beta3 subunit 825T allele and hypertension. Hypertension 34: e8.

Siffert W, Rosskopf D, Moritz A, Wieland T, Kaldenberg-Stasch S, Kettler $\mathrm{N}$ et al (1995). Enhanced $\mathrm{G}$ protein activation in immortalized lymphoblasts from patients with essential hypertension. J Clin Invest 96: 759-766.
Stahl SM (1998). Basic psychopharmacology of antidepressants, part 1: Antidepressants have seven distinct mechanisms of action. J Clin Psychiatry 59(Suppl 4): 5-14.

Yoshida K, Naito S, Takahashi H, Sato K, Ito K, Kamata M et al (2002). Monoamine oxidase: A gene polymorphism, tryptophan hydroxylase gene polymorphism and antidepressant response to fluvoxamine in Japanese patients with major depressive disorder. Prog Neuropsychopharmacol Biol Psychiatry 26: $1279-1283$. 